\title{
Joint crisis plans for people with borderline personality disorder: feasibility and outcomes in a randomised controlled trial
}

Rohan Borschmann, Barbara Barrett, Jennifer M. Hellier, Sarah Byford, Claire Henderson, Diana Rose, Mike Slade, Kim Sutherby, George Szmukler, Graham Thornicroft, Joanna Hogg and Paul Moran

\section{Background}

People with borderline personality disorder frequently experience crises. To date, no randomised controlled trials (RCTS) of crisis interventions for this population have been published.

\section{Aims}

To examine the feasibility of recruiting and retaining adults with borderline personality disorder to a pilot RCT investigating the potential efficacy and cost-effectiveness of using a joint crisis plan.

\section{Method}

An RCT of joint crisis plans for community-dwelling adults with borderline personality disorder (trial registration: ISRCTN12440268). The primary outcome measure was the occurrence of self-harming behaviour over the 6-month period following randomisation. Secondary outcomes included depression, anxiety, engagement and satisfaction with services, quality of life, well-being and costeffectiveness.

\section{Results}

In total, 88 adults out of the 133 referred were eligible and were randomised to receive a joint crisis plan in addition to treatment as usual (TAU; $n=46$ ) or TAU alone $(n=42)$. This represented approximately $75 \%$ of our target sample size and follow-up data were collected on 73 (83.0\%) participants. Intention-to-treat analysis revealed no significant differences in the proportion of participants who reported self-harming (odds ratio $(\mathrm{OR})=1.9,95 \% \mathrm{Cl} 0.53-6.5, P=0.33$ ) or the frequency of self-harming behaviour (rate ratio (RR) $=0.74,95 \%$ Cl 0.34-1.63, $P=0.46$ ) between the two groups at follow-up. No significant differences were observed between the two groups on any of the secondary outcome measures or costs.

\section{Conclusions}

It is feasible to recruit and retain people with borderline personality disorder to a trial of joint crisis plans and the intervention appears to have high face validity with this population. However, we found no evidence of clinical efficacy in this feasibility study.

\section{Declaration of interest}

None.
People with borderline personality disorder experience considerable instability in their emotions and relationships with others, and this can lead to frequent crises and acts of self-harm. ${ }^{1}$ Self-harm is the strongest predictor of completed suicide ${ }^{2}$ and, as such, the consequences of people with borderline personality disorder not receiving appropriate interventions during times of crises are potentially lethal. Despite this, relatively little research has examined the management of acute crises for people with borderline personality disorder. ${ }^{3} \mathrm{~A}$ joint crisis plan (JCP) is a written document containing a mental health service user's treatment preferences for the management of future crises. ${ }^{4}$ The service user develops the JCP in collaboration with their treating clinician at a meeting that is facilitated by an independent mental health practitioner. Although the main aim of a JCP is to enhance the service user's empowerment regarding their own care, other benefits, such as reduced levels of mental health service use, reduced levels of perceived coercion and enhanced therapeutic alliance, may also be achieved. ${ }^{4}$ Previous research has found JCPs to be an effective way of reducing coercive treatment for people with psychosis. ${ }^{5}$ However, there have been no published randomised controlled trials (RCTs) investigating the impact of personalised crisis plans for people with borderline personality disorder. ${ }^{3}$ We conducted a pilot RCT, the aims of which were to investigate (a) the feasibility of recruiting and retaining a sample of community-dwelling adults with borderline personality disorder to a trial of JCPs and (b) the potential efficacy and cost-effectiveness of using a JCP on the self-harming behaviour of participants.

\section{Method}

\section{Trial design and participants}

We undertook a parallel group, single blind, treatment as usual (TAU)-controlled, randomised trial of participants with borderline personality disorder. We specifically wanted to recruit participants who experienced crises and engaged in self-harming behaviour. Follow-up was considered at 6-months post-randomisation. We recruited a sample of adults accessing community mental health teams (CMHTs) in south east London, UK. Inclusion criteria were:

(a) aged 18 years or older;

(b) meeting diagnostic criteria for borderline personality disorder (according to DSM-IV-TR criteria ${ }^{6}$ and measured using the Structured Clinical Interview for DSM-IV (SCID-II) - Borderline Personality Disorder subsection); ${ }^{7}$

(c) had self-harmed in the previous 12 months (defined as at least one act with a non-fatal outcome in which the individual had initiated a behaviour (such as self-cutting), or ingested a toxic substance or object, with the intention of causing harm to themselves) ${ }^{8}$

(d) under the ongoing care of a CMHT;

(e) able to provide written informed consent.

Exclusion criteria were:

(a) currently an in-patient;

(b) primary diagnosis of a psychotic illness; 
(c) unable to read or write in English;

(d) unable to provide written informed consent.

All potential participants were in the first instance identified and approached by their care coordinator, who informed them about the trial. After they had expressed an interest in participating, a member of the research team met with the participant to explain the trial further and obtain written informed consent.

\section{Ethics and governance approvals}

Data collection protocols were approved by the South London Research Ethics Committee (ref: 09/H0803/113) and the trial was registered with the International Standard Randomised Controlled Trial registry (ISRCTN12440268) prior to the commencement of data collection. ${ }^{9}$ All participants provided written informed consent prior to entering the trial, including allowing members of the research team to access their electronic records. Progress of the trial, adherence to protocol and participant safety were overseen by a trial steering committee (chaired by Professor Mike Crawford, Imperial College London).

\section{Randomisation and masking}

After consent and baseline assessment, randomisation was conducted at the level of the individual and was stratified by alcohol use (as measured by scores on the Alcohol Use Disorders Identification Test (AUDIT); ${ }^{10}$ low $<8$; medium 8-15; high $>15$ ) and depression (as measured by scores on the Hospital Anxiety and Depression Scale (HADS) ${ }^{11}$ depression subscale; low $<8$, medium $8-10$, high $>10$ ), both of which have been shown to be predictive of future self-harm. ${ }^{12,13}$ Randomisation was managed electronically by the Clinical Trials Unit at the King's College London Institute of Psychiatry, UK. The nature of the intervention meant that neither participants nor staff members could be masked to allocation; however, all follow-up data were collected by a research worker who was masked to treatment allocation and all data analyses were conducted by a statistician who was also masked to treatment allocation. The extent to which masking was achieved in the collection of outcome data was assessed at the end of the trial.

\section{Intervention and control arms}

\section{Joint crisis plan}

Participants randomised to the JCP + TAU condition were provided with a blank template of a JCP that included a list of topics to be considered for inclusion in the participant's JCP. Topics included 'Positive things I can do in a crisis', 'Specific refusals regarding treatment during a crisis', 'Practical help in a crisis' and 'Useful telephone numbers'. A fictional JCP is displayed in online supplement DS1. Approximately 1 week later, a JCP planning meeting between the participant and their care coordinator was arranged (and facilitated) by R.B. Other key workers, advocates, friends or family members were also invited at the discretion of the participant. The aim of this meeting was to have a facilitated, informed discussion about the most appropriate information to be included in the participant's JCP. Meetings lasted approximately $60 \mathrm{~min}$ and the final information included in the JCP was of the participant's choosing and was entered in the participant's own wording. Within $24 \mathrm{~h}$ of the meeting, a typed version of the JCP was distributed to all individuals specified by the participant. With the participant's permission (56.1\% of participants consented), a copy of the JCP was also attached to their electronic medical records in order to maximise dissemination of the plan within the local mental health trust.

\section{Treatment as usual}

Participants in both groups continued to receive standard care from their treating CMHT. This included, as a part of the care programme approach (CPA), the provision for service users to receive written copies of their care plan, including a brief 'crisis contingency plan', in addition to regular contact with a care coordinator or allocated member of the clinical team.

\section{Outcome measures}

The primary outcome was the proportion of participants reporting self-harm at 6 months post-randomisation. Self-harm data were obtained from an established self-report questionnaire. ${ }^{14}$ Items included 'How many times in the past year [or 'past six months' at follow-up] have you deliberately tried to harm yourself?' Secondary clinical outcomes, all measured at baseline and follow-up, and their corresponding instruments were as follows.

(a) Depression and anxiety: HADS. ${ }^{11}$ This is a 14 -item self-report scale for detecting states of depression and anxiety in outpatients, with higher scores indicating higher levels of depression/anxiety.

(b) Working alliance: Working Alliance Inventory (WAI). ${ }^{15}$ The WAI is a 12-item self-report instrument for measuring the perceived quality of working alliance between client and practitioner, with higher scores indicating a more positive perception of alliance.

(c) Satisfaction with services: Client Satisfaction Questionnaire (CSQ). ${ }^{16}$ The CSQ is an eight-item measure of participants' level of satisfaction with treatment received, with higher scores indicating a higher level of satisfaction with services.

(d) Engagement with services: Service Engagement Scale (SES). ${ }^{17}$ The SES is a 14-item self-report scale, completed by the participant's treating clinician - in our trial typically a care coordinator or key worker - to measure the participant's level of engagement with community mental health services. Higher scores reflect a greater level of difficulty engaging with services.

(e) Well-being: Warwick-Edinburgh Mental Well-Being Scale (WEMWBS). ${ }^{18}$ The WEMWBS is a measure of subjective mental well-being over the preceding 2 weeks and focuses entirely on positive aspects of mental health. Higher scores indicate a higher level of well-being.

(f) Social functioning: Work and Social Adjustment Scale (WSAS). ${ }^{19}$ The WSAS is a five-item self-report instrument to assess impaired functioning, with higher scores indicating a higher level of impairment.

(g) Perceived coercion: Treatment Experience Scale (TES). The TES was adapted from the Admission Experience Survey, ${ }^{20}$ a 16-item instrument designed to assess the perceived level of coercion experienced by service users during hospital admission. Respondents endorse each item as either 'true', 'false' or 'don't know'.

(h) Health-related quality of life: EuroQoL 5-dimensions (EQ-5D). ${ }^{21}$ The EQ-5D assesses respondents' subjective health-related quality of life across five life domains: mobility, self-care, usual activities, pain/discomfort and anxiety/depression. A higher score indicates a better health-related quality of life.

(i) Resource-use: Adult Service Use Schedule (AD-SUS) adapted for use in this trial based on previous research involving people with personality disorders. 22 The AD-SUS, completed by participants in interview at baseline and 6-month followup, collected data on use of all hospital and community 
health and social services. To enhance accuracy, in-patient psychiatric admission data were additionally collected from electronic clinical records of the local National Health Service (NHS) trust (South London and Maudsley). This data replaced self-reported contact data for this NHS trust, although self-reported contacts with other trusts were retained. The economic evaluation took a health and social care perspective, in line with National Institute for Health and Clinical Excellence guidelines. ${ }^{23}$ Unit costs for the financial year 2009-2010 were applied, and these are detailed in online supplement DS2.

\section{Sample size and power calculation}

Sample size calculations are not required for most pilot studies, because the aim is to gather information about recruitment processes, consent and attrition rates and trial procedures. Nevertheless, we wanted to know whether it was feasible to recruit and retain a pre-determined number of people with borderline personality disorder into a trial of JCPs and, for this reason, we undertook a power calculation in order to give us a target sample size to aim for. The trial was powered to detect a threefold difference in the proportions of participants who had self-harmed during the follow-up period (36\% in the TAU group $v .12 \%$ in the JCP + TAU group). The predicted TAU proportion of self-harm $(36 \%)$ was the same proportion of self-harm as that observed in a previous RCT of cognitive therapy to reduce repetition of selfharm. ${ }^{24}$ On the basis of these figures, an overall sample of 114 (randomised 1:1 to JCP + TAU:TAU) would provide $80 \%$ power to detect an observed difference between JCP + TAU and TAU alone, based on a two-sided test at the 5\% significance level. The target sample size was increased to 120 in order to allow for attrition and loss of data on self-harm. This sample would also be large enough to provide $80 \%$ power to detect a constant hazard ratio between the groups of 0.29 with proportions of episodes in the two groups as stated above, based on the log-rank statistic assuming no accrual rate, a fixed time of follow-up and an estimated $10 \%$ rate of drop out.

\section{Statistical analyses}

All analyses were based on the intention-to-treat sample using a statistical analysis plan finalised by the trial statistician (J.M.H.) and approved by the principal investigator (P.M.) in advance of conducting any analyses. All analyses were performed with Stata version 11.0 for Windows.

\section{Analysis of outcome variables}

We summarised continuous variables as mean (s.d.) and categorical variables as $n(\%)$. We assessed the primary outcome with a logistic regression model with treatment and stratification factors; alcohol misuse (AUDIT) and depression (HADS) as covariates. Model assumptions were checked by the use of diagnostic plots. Models were undertaken with the assumption that data were missing at random. Categorical data were compared using Fisher's Exact test. We analysed secondary outcomes in a generalised linear model (GLM) framework; covariates in the model were treatment group, baseline value of outcome, alcohol misuse and depression. For the frequency of self-harm at 6-month follow-up, a negative binomial distribution was specified with a log link. Logistic regression was utilised for binary outcomes and clinical scales were analysed using the assumption of a normal distribution. Results of the treatment effects were summarised as odds ratios (ORs, logistic and ordinal logistic regression), incidence rate ratios (RRs, negative binomial distribution GLM) and effect sizes
(Gaussian models) at 6-month follow-up with two-sided 95\% confidence intervals.

\section{Analysis of cost data}

Differences in the use of services between randomised groups were compared descriptively and no statistical comparisons were made. Total cost per participant over the 6-month follow-up was calculated and compared statistically. Although costs were not normally distributed, analysis compared mean costs between the two randomised groups using standard parametric tests, as recommended for the analysis of cost data, ${ }^{25}$ with the robustness of the parametric tests confirmed using bias-corrected, nonparametric bootstrapping. ${ }^{26}$ Baseline cost and stratification variables (alcohol misuse and depression) were included as covariates.

\section{Cost-effectiveness analysis}

For this feasibility study, cost-effectiveness was primarily explored descriptively, taking a cost-consequences approach, which involves the presentation of a range of outcome measures alongside the costs. In cost-consequences analysis, no attempt is made to combine the costs and effectiveness of alternative interventions and decision makers are left to form their own opinion regarding the relative importance of the alternative outcomes presented. A formal cost-effectiveness analysis was carried out, as detailed in our original protocol; ${ }^{9}$ however, given the small sample sizes involved, this was considered hypothesisgenerating only and is reported in online supplement DS2 for information.

\section{Results}

\section{Feasibility of recruitment}

Two full-time research workers recruited 88 participants over 17 months. In total, 133 individuals were referred; 30 declined and 3 were unable to read or write in English (both being necessary for participation in the formulation of a written crisis plan). Of the remaining 100 consenting individuals, 12 were ineligible (7 did not meet diagnostic criteria for borderline personality disorder and 5 had not self-harmed in the previous 12 months); the final sample, therefore, consisted of 88 participants (Fig. 1). A total of 46 participants were randomised to the JCP + TAU arm and 42 were randomised to the TAU arm. We obtained complete follow-up data on $73(83.0 \%)$ participants; $37(80.4 \%)$ from the JCP + TAU arm and $36(85.7 \%)$ from the TAU arm.

\section{Feasibility of the baseline assessments}

All 88 participants completed the full battery of assessments at baseline, with the exception of the WEMWBS, which was added to the battery, on the advice of the project advisory group, after 48 participants had entered the study. Consequently, only 40 participants $(45.4 \%)$ completed this measure at baseline. All assessment sessions lasted between 45 and $90 \mathrm{~min}$.

\section{Baseline characteristics}

Table 1 shows the participant demographics recorded at baseline. The majority of participants were White British, female, aged in their 30s, single and unemployed. Most had left school prior to the age of 16. Demographic data were evenly matched across the TAU and JCP + TAU arms, reflecting the effectiveness of the stratified randomisation process. 


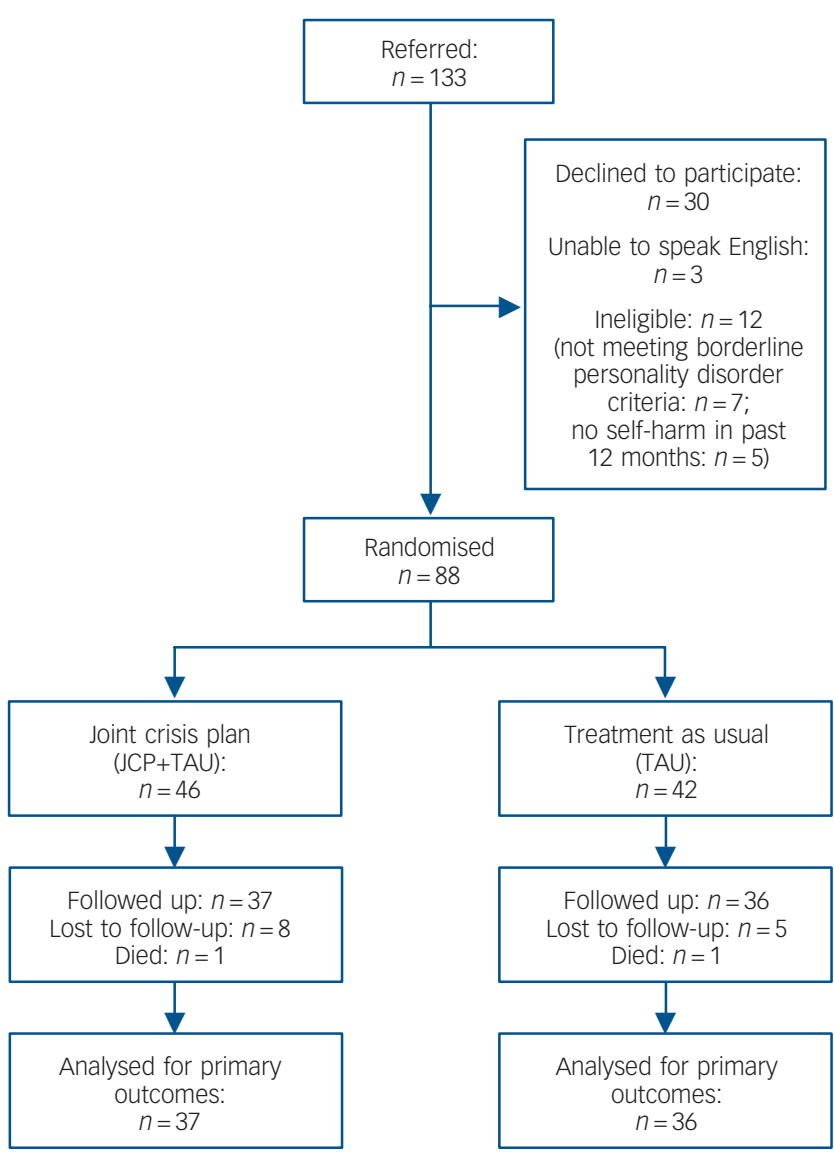

Fig. 1 Trial CONSORT diagram.

\section{Attrition}

Thirteen participants $(14.7 \%)$ dropped out of the trial prior to follow-up; $8 / 46(17.4 \%)$ from the JCP + TAU group and 5/42 (11.9\%) from the TAU group. This figure was higher than the $10 \%$ we had estimated and contributed to the trial being underpowered. Additionally, there were two serious adverse events as two participants (one from each trial arm) died during the follow-up period; neither of these deaths was related to the intervention.

\section{Feasibility of the follow-up assessments}

In total 73 participants completed the battery of assessments at follow-up and, because of the shorter battery length, assessment sessions lasted between 30 and $60 \mathrm{~min}$. All outcome measures were completed by between 63 and 73 participants (71.6-82.9\%) at follow-up.

\section{Self-harm}

Table 2 shows the mean number of self-harm episodes reported by participants in each arm of the trial, along with the dichotomised self-harm data at baseline and 6-month follow-up. In both arms, at 6-month follow-up, the proportion of participants reporting self-harm had fallen. However, there was no significant difference in the proportion reporting self-harm between the JCP + TAU and TAU arms $(\mathrm{OR}=1.9,95 \% \mathrm{CI} 0.53-6.5, P=0.33)$. There were also no significant differences in the number of self-harm acts reported between the two groups $(\mathrm{RR}=0.74,95 \% \mathrm{CI} 0.34-1.63, P=0.46)$.
Table 1 Baseline demographics and stratification of study participants

\begin{tabular}{|c|c|c|c|}
\hline Variable & $\begin{array}{l}\text { Treatment } \\
\text { as usual } \\
(n=42)\end{array}$ & $\begin{array}{l}\text { Joint crisis } \\
\text { plans }+ \\
\text { treatment as } \\
\text { usual }(n=46)\end{array}$ & $\begin{array}{l}\text { Total } \\
(n=88)\end{array}$ \\
\hline \multicolumn{4}{|l|}{$\begin{array}{l}\text { Alcohol, Alcohol Use Disorders } \\
\text { Identification Test score: } n(\%)\end{array}$} \\
\hline$<8$ & $20(47.6)$ & 19 (41.3) & $39(44.3)$ \\
\hline $8-15$ & 5 (11.9) & $9(19.6)$ & $14(15.9)$ \\
\hline$>15$ & $17(40.5)$ & $18(39.1)$ & $35(39.8)$ \\
\hline \multicolumn{4}{|l|}{$\begin{array}{l}\text { Depression, Hospital Anxiety } \\
\text { and Depression Scale } \\
\text { (depression subscale): } n \text { (\%) }\end{array}$} \\
\hline$<8$ & $6(14.3)$ & $8(17.4)$ & $14(15.9)$ \\
\hline $8-10$ & $10(23.8)$ & $6(13.0)$ & $16(18.2)$ \\
\hline$>10$ & $26(61.9)$ & $32(69.6)$ & $58(65.9)$ \\
\hline $\begin{array}{l}\text { Age at randomisation, years: } \\
\text { mean s.d) }\end{array}$ & 36.1 (12.37) & $35.6(11.1)$ & $35.8(11.6)$ \\
\hline Male, $n(\%)$ & $7(16.7)$ & $10(21.7)$ & $17(19.3)$ \\
\hline \multicolumn{4}{|l|}{ Relationship status, $n$ (\%) } \\
\hline In a relationship & $5(11.9)$ & $8(17.4)$ & $13(14.8)$ \\
\hline Not in a relationship & $37(88.1)$ & $38(82.6)$ & $75(85.2)$ \\
\hline \multicolumn{4}{|l|}{ Living status, $n$ (\%) } \\
\hline Alone & $20(47.6)$ & $22(47.8)$ & $42(47.7)$ \\
\hline With other(s) & $19(45.2)$ & $20(43.5)$ & 39 (44.3) \\
\hline Supervised/assisted living & $3(7.1)$ & $4(8.7)$ & $7(8.0)$ \\
\hline \multicolumn{4}{|l|}{ Ethnicity, $n(\%)$} \\
\hline Asian & $1(2.4)$ & $0(0.0)$ & $1(1.1)$ \\
\hline Black & $3(7.1)$ & $6(13.0)$ & $9(10.2)$ \\
\hline White & $31(73.8)$ & $34(73.9)$ & 65 (73.9) \\
\hline Mixed & $4(9.5)$ & $3(6.5)$ & $7(8.0)$ \\
\hline Other & $3(7.1)$ & $3(6.5)$ & $6(6.8)$ \\
\hline \multicolumn{4}{|l|}{ Employment status, $n$ (\%) } \\
\hline In paid work & $4(9.5)$ & $6(13.0)$ & $10(11.4)$ \\
\hline Not working & $16(38.0)$ & $20(43.5)$ & $36(40.9)$ \\
\hline Permanently sick or disabled & $22(52.4)$ & $20(43.5)$ & $42(47.7)$ \\
\hline \multicolumn{4}{|l|}{ Age left school, years } \\
\hline Mean (s.d.) & $15.9(1.3)$ & $15.8(1.4)$ & $15.9(1.3)$ \\
\hline Range (minimum to maximum) & $12-18$ & $10-19$ & 10-9 \\
\hline Further education, yes: $n$ (\%) & $30(71.4)$ & $31(67.4)$ & $61(69.3)$ \\
\hline \multicolumn{4}{|l|}{ Site, $n(\%)$} \\
\hline Lambeth & $7(16.7)$ & $4(8.7)$ & $11(12.5)$ \\
\hline Southwark & $17(40.5)$ & $18(39.1)$ & $35(39.8)$ \\
\hline Lewisham & $3(7.1)$ & 7 (15.2) & $10(11.4)$ \\
\hline Croydon & 14 (33.3) & $10(21.7)$ & $24(27.3)$ \\
\hline Greenwich & $1(2.4)$ & $7(15.2)$ & $8(9.1)$ \\
\hline
\end{tabular}

\section{Use of JCPS}

Of the 46 participants allocated to receiving JCP, 41 (89.1\%) attended their JCP planning meeting. Of these, $34(82.9 \%$ of JCP + TAU participants) were available for follow-up (along with 3 participants who did not attend their JCP meeting). In total, $25(73.5 \%)$ of these individuals reported using their JCP during a crisis and $15(44.1 \%)$ reported using it between crises. Almost half $(n=16,47.1 \%)$ reported that using their JCP had contributed to having a greater feeling of control over their problems and $47.1 \%$ reported that it had contributed to an improved relationship with their mental health team. Twenty-nine (85.2\%) stated that they would recommend using a JCP to other service users.

\section{Resource use and costs}

The mean number of contacts participants had with all health, social care and criminal justice sector services over the 6-month follow-up period is detailed in Table 3. Mean costs per participant over the 6-month follow-up period are detailed in Table 4. The 


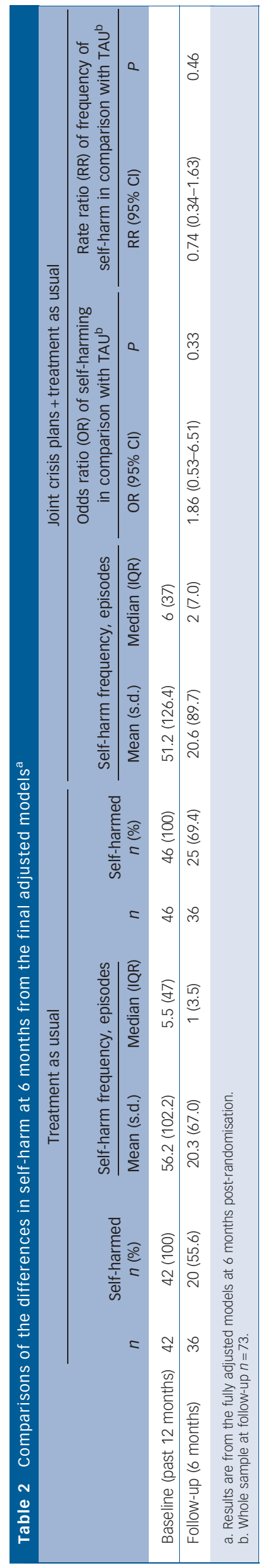

average cost of the JCP intervention was estimated to be $£ 146$ per participant. There were no significant differences in total health and social care costs (mean cost $£ 5631$ TAU $v$. $£ 5308$ JCP + TAU, $P=0.20$ ). Sensitivity analyses exploring the impact of missing data and the cost of JCPs (available from the author on request) did not alter these findings.

\section{Secondary outcomes}

Table 5 shows the secondary outcomes reported at baseline and follow-up by participants in the two trial arms. Randomisation was stratified by AUDIT (alcohol) and HADS (depression) scores; at baseline, participants recorded a mean depression score of 11.77 (s.d. $=4.64$ ) from a possible score of 21 , reflecting the presence of moderate depressive symptoms across the sample. Participants recorded a mean alcohol score of $13.2($ s.d. $=12.0)$ from a possible score of 40, placing the average participant into the 'moderately problematic' category of alcohol consumption. ${ }^{10}$ There were no significant differences between the groups on any of the secondary outcome measures at follow-up.

\section{Discussion}

\section{Main findings}

Our study revealed that it is feasible to recruit and retain people with borderline personality disorder to a clinical trial of JCPs. We recruited approximately three-quarters of our target sample size and retained more than $80 \%$ of participants through the trial. Moreover, the intervention appeared to have high face validity with the trial participants. Although other borderline personality disorder intervention studies have included a crisis management component as one of the ingredients of treatment, ${ }^{27,28}$ to our knowledge, this is the first RCT of a crisis intervention specifically tailored to people with borderline personality disorder. The JCPs were used both during and between crises and were viewed favourably by participants. Approximately half of participants reported a greater sense of control over their problems and an improved relationship with their mental health team when using a JCP and the large majority of participants stated that they would recommend using a JCP to other service users. At follow-up, the proportion of participants reporting self-harm fell in both groups. However, there was no significant difference in the proportions reporting self-harm between groups, and no significant differences between the groups on any of the secondary outcome measures. In addition, we did not detect a significant health and social care cost difference between the two groups over the 6-month follow-up, although there was some suggestion of greater service use in the TAU group.

\section{Possible explanations for the findings}

The lack of statistically significant differences between the groups on primary or secondary outcomes in the face of high user acceptability is counter-intuitive. However, we may have failed to detect significant differences between the two groups for a number of reasons. First, crisis planning for people with borderline personality disorder may be more successful when the crisis plan is fully integrated with other components of treatment, ${ }^{29}$ as opposed to the one-off intervention offered to participants in the experimental arm of this trial. Second, we underrecruited to the study and it was therefore underpowered to detect a significant difference on the primary outcome. Recruitment was more difficult and attrition higher than we had originally anticipated when designing the trial. These findings emphasise the need to allow for a longer recruitment phase and 
Table 3 Mean use of health and social care services over 6-month follow-up

\begin{tabular}{|lcc|} 
& \multicolumn{2}{c|}{ Mean (s.d.) } \\
\cline { 2 - 3 } & $\begin{array}{c}\text { Treatment as } \\
\text { usual }(n=36)\end{array}$ & $\begin{array}{c}\text { Joint crisis } \\
\text { plans + treatment } \\
\text { as usual }(n=37)\end{array}$ \\
\hline In-patient mental health nights & $4.3(17.2)$ & $6.1(12.2)$ \\
\hline In-patient physical health nights & $0.2(0.9)$ & $0.3(1.0)$ \\
\hline Out-patient appointments & $7.5(20.4)$ & $4.4(7.7)$ \\
\hline Accident and emergency attendances & $1.3(3.0)$ & $2.1(5.9)$ \\
\hline General practitioner contacts & $9.0(10.1)$ & $6.2(7.2)$ \\
\hline Community mental health contacts & $32.2(51.8)$ & $22.5(18.9)$ \\
\hline Community healthcare contacts & $3.3(6.2)$ & $1.8(6.0)$ \\
\hline Community advice contacts & $5.7(16.4)$ & $3.6(6.5)$ \\
\hline
\end{tabular}

larger inflation factors in the calculation of sample size for trials involving people with borderline personality disorder. Less than a third of publicly funded trials manage to recruit according to their original plan ${ }^{30}$ and so the recruitment difficulties we experienced may also have reflected a current wider problem of underrecruitment of NHS patients into research studies. Third, some participants in the TAU group may have received a generic, but equally efficacious, crisis contingency plan as part of their concurrent TAU under the CPA. However, a 2007 audit of South London and Maudsley Trust service users who had attended the emergency department (followed up 9 months later) revealed that $42 \%$ of those under the standard CPA did not have a crisis contingency plan on their electronic records (unpublished data). Of those that did, only $37 \%$ of plans contained any information that was specific to the service user, with the remaining plans consisting solely of generic information. It seems unlikely,

Table 4 Total health and social care cost per participant over 6-month follow-up

\begin{tabular}{|c|c|c|c|c|}
\hline & \multicolumn{2}{|c|}{ Mean (s.d.), $f$} & \multirow[b]{2}{*}{$\begin{array}{c}\text { Mean difference } \\
(95 \% \mathrm{Cl} \text {, bootstrapped })^{\mathrm{a}}\end{array}$} & \multirow[b]{2}{*}{$P$} \\
\hline & $\begin{array}{l}\text { Treatment as usual } \\
\qquad(n=36)\end{array}$ & $\begin{array}{c}\text { Joint crisis plans + treatment } \\
\text { as usual }(n=37)\end{array}$ & & \\
\hline Joint crisis plan & $0(0)$ & $146(0)$ & & \\
\hline Hospital & $2690(8083)$ & 2761 (3919) & & \\
\hline Community health and social care & 2255 (3158) & 2115 (2819) & & \\
\hline Medication & $447(887)$ & $260(497)$ & & \\
\hline Criminal justice sector services & $238(664)$ & $26(90)$ & & \\
\hline Total service costs & 5631 (10293) & $5308(5486)$ & $-324(-6369-2034)$ & 0.20 \\
\hline
\end{tabular}

Table 5 Secondary clinical outcomes reported at baseline and follow-up by participants in the treatment as usual and treatment as usual + joint crisis plans arms ${ }^{a}$

\begin{tabular}{|c|c|c|c|c|}
\hline \multirow[b]{2}{*}{ Clinical scale (range) } & \multicolumn{2}{|c|}{ Treatment as usual } & \multicolumn{2}{|c|}{ Joint crisis plans + treatment as usual } \\
\hline & $n$ & Mean (s.d.) & $n$ & Mean (s.d.) \\
\hline \multicolumn{5}{|c|}{ Highest score is best outcome } \\
\hline \multicolumn{5}{|c|}{ Working Alliance Inventory Client (WAI-C) (12-84) } \\
\hline Baseline & 33 & $63.36(17.92)$ & 38 & $58.47(18.50)$ \\
\hline Month 6 & 30 & $60.47(15.92)$ & 33 & $58.85(16.75)$ \\
\hline \multicolumn{5}{|c|}{ Working Alliance Inventory Therapist (WAI-T) (12-84) } \\
\hline Baseline & 37 & $61.27(11.10)$ & 40 & $63.68(8.72)$ \\
\hline Month 6 & 25 & $62.96(10.74)$ & 29 & $64.66(10.87)$ \\
\hline \multicolumn{5}{|c|}{ Client Satisfaction Questionnaire (CSQ) (4-32) } \\
\hline Baseline & 37 & $18.62(1.53)$ & 41 & $19.85(1.46)$ \\
\hline Month 6 & 36 & $19.64(1.33)$ & 37 & $19.97(2.0)$ \\
\hline \multicolumn{5}{|c|}{ Warwick-Edinburgh Mental Well-Being Scale (WEMWBS) (14-70) } \\
\hline Baseline & 23 & $31.74(10.14)$ & 26 & $29.65(11.09)$ \\
\hline Month 6 & 35 & $35.26(10.26)$ & 36 & $34.33(11.40)$ \\
\hline \multicolumn{5}{|c|}{ Lowest score is best outcome } \\
\hline \multicolumn{5}{|c|}{ Work and Social Adjustment Scale (WSAS) (0-40) } \\
\hline Baseline & 42 & $26.95(7.36)$ & 46 & $27.02(6.46)$ \\
\hline Month 6 & 36 & $26.06(7.98)$ & 36 & $25.81(8.94)$ \\
\hline \multicolumn{5}{|c|}{ Treatment Experience Scale (TES) (0-45) } \\
\hline Baseline & 42 & $16.52(2.75)$ & 46 & $17.04(2.97)$ \\
\hline Month 6 & 36 & $16.0(3.07)$ & 37 & $17.68(3.09)$ \\
\hline \multicolumn{5}{|c|}{ Hospital Anxiety and Depression Scale - Depression (HADS-D) (0-21) } \\
\hline Baseline & 42 & $11.76(4.30)$ & 46 & $11.78(4.98)$ \\
\hline Month 6 & 34 & $10.47(3.54)$ & 35 & $10.20(4.96)$ \\
\hline \multicolumn{5}{|c|}{ Hospital Anxiety and Depression Scale - Anxiety (HADS-A) (0-21) } \\
\hline Baseline & 42 & $14.48(5.55)$ & 46 & $14.46(4.07)$ \\
\hline Month 6 & 36 & $12.94(4.55)$ & 37 & $14.57(3.83)$ \\
\hline \multicolumn{5}{|c|}{ Service Engagement Scale (SES) (0-42) } \\
\hline Baseline & 34 & $10.41(7.14)$ & 38 & $9.82(6.04)$ \\
\hline Month 6 & 25 & $10.88(5.62)$ & 30 & $8.63(6.11)$ \\
\hline
\end{tabular}


therefore, that such generic crisis contingency plans (which are written by the clinician, without input from the service user), would have contributed to the absence of a significant difference between intervention groups in this study.

\section{Strengths and limitations}

In addition to the problem of underrecruitment, the study had other limitations. First, we relied exclusively on self-report for the collection of data on self-harm. Similar self-report methods have been used in previous RCTs aiming to reduce self-harming behaviour. $^{31}$ However, there is an inherent risk associated with using this methodology for obtaining self-harm data, as it is dependant entirely on respondents' openness and comprehension of questionnaire items. ${ }^{32}$ It may also be susceptible to reporting bias (unintentional or otherwise) and the occurrence of both false negatives and false positives is possible. Additionally, participant recall at 6 months may not have been accurate ${ }^{33}$ and this may have had an impact on our findings. Second, with the exception of the most recent act of self-harm, we did not measure the medical severity of participants' self-harm and neither did we enquire about their behavioural intention. Given that such intentions can vary considerably between individuals and even within the same individual at different times, these may have been important data to collect.

Third, 'treatment as usual' for people with borderline personality disorder varies greatly between CMHTs, between clinicians and between individual service users. Some participants in our trial reported not seeing their CMHT care coordinator at all during the 6-month follow-up period (despite still being registered as an active service user), whereas others reported being in contact with their care coordinator several times each week during the same period. The net result of this was that participants allocated to the TAU arm received considerable variation in treatment, although the impact of this on our findings is difficult to assess. Finally, our follow-up period was limited to 6 months and it is possible that a longer period of follow-up may have resulted in significant clinical change.

Our trial also had several strengths. The trial was conducted in a real NHS setting, with recruitment taking place across five separate and demographically disparate boroughs during a period of considerable austerity and restructuring of services. Despite this, we recruited to approximately $75 \%$ of our target sample size and retained more than $80 \%$ at 6 -month follow-up. Second, our refusal rate of $25 \%$ was comparable with those reported in other RCTs involving patients with borderline personality disorder ${ }^{31,34}$ and our response rate of $75 \%$ was twice as high as that reported in a previous large-scale RCT of JCPs. ${ }^{5}$ Third, the stratified randomisation process was effective, as evidenced by the similarity of the intervention and control groups in both size and demographic characteristics. Fourth, although the trial could not have utilised a double-blind methodology, all data analyses were conducted masked to treatment allocation and follow-up data were collected by a researcher masked to treatment allocation and this masking was maintained in 62 of 73 cases (85\%). Finally, a total of 41 out of 46 participants in the JCP + TAU group (89\%) received the active intervention, reflecting high fidelity to the intervention.

\section{Clinical implications}

Previous research using JCPs and other psychiatric advance directives has found that such plans promote self-determination and empowerment among service users ${ }^{35}$ and that they have the potential to facilitate stronger relationships between service users and providers. ${ }^{36}$ Fostering collaborative relationships is essential in the treatment of people with borderline personality disorder ${ }^{1}$ and JCPs may provide one approach to ensuring that the values and treatment preferences of such individuals remain central when they experience crises. Although we found that JCPs have high face validity for people with borderline personality disorder, we did not find evidence of clinical efficacy and so our trial does not provide justification to recommend the use of JCPs in clinical practice. However, as our trial was underpowered, it remains possible that the JCP is an effective intervention for people with borderline personality disorder. Future investigation will need to include robust process evaluation to understand why the experience of receiving this intervention was so positive.

Rohan Borschmann, DclinPsy, Barbara Barrett, PhD, Health Services \& Population Research Department, Institute of Psychiatry, King's College London; Jennifer M. Hellier, MSc, King's Clinical Trials Unit, Department of Biostatistics, Institute of Psychiatry, King's College London; Sarah Byford, PhD, Claire Henderson, PhD, MRCPsych, Diana Rose, PhD, Mike Slade, PhD, PsychD, Kim Sutherby, MRCPsych, George Szmukler MD, FRCPSych, Graham Thornicroft, PhD, FRCPSych, Joann Hogg, MSc, Paul Moran, MD, MRCPsych, Health Services \& Population Research Department, Institute of Psychiatry, King's College London, UK

Correspondence: Paul Moran, PO28, David Goldberg Building, Institute of Psychiatry, King's College London, De Crespigny Park, London SE5 8AF, UK. Email: paul.moran@kcl.ac.uk

First received 13 Jul 2012, final revision 14 Feb 2012, accepted 18 Feb 2013

\section{Funding}

This trial was supported by a Medical Research Council (MRC) trial platform grant (ID: 85397) in the UK. P.M., G.T. and D.R. are part-funded by the NIHR Specialist Biomedical Research Centre for Mental Health at the South London and Maudsley NHS Foundation Trust and Kings College London.

\section{Acknowledgements}

We are grateful to all trial participants and to the external members of the trial steering committee: Professor Mike Crawford (Chair) and Dr Sue Patterson from Imperial College London and Victoria Green from the University of Leeds. We also thank the King's Clinical Trials Unit for provision of the InferMed MACRO data entry system and web-based randomisation system for the trial.

\section{References}

1 National Institute for Health and Clinical Excellence. Borderline Personality Disorder: Treatment and Management. NICE Clinical Guideline 78. NICE, 2009.

2 Cooper J, Kapur N, Webb R, Lawlor M, Guthrie E, Mackway-Jones K, et al. Suicide after deliberate self-harm: a 4-year cohort study. Am J Psychiatry 2005; 162: 297-303

3 Borschmann R, Henderson C, Hogg J, Phillips R, Moran P. Crisis interventions for people with borderline personality disorder. Cochrane Database Syst Rev 2012; 6: CD009353.

4 Henderson C, Swanson JW, Szmukler G, Thornicroft G, Zinkler M. A typology of advance statements in mental health care. Psychiatr Serv 2008; 59: 63-71.

5 Henderson C, Flood C, Leese M, Thornicroft G, Sutherby K, Szmukler G. Effect of joint crisis plans on use of compulsory treatment in psychiatry: single blind randomized controlled trial. BMJ 2004; 329: 126-30.

6 American Psychiatric Association. Diagnostic and Statistical Manual of Mental Disorders (4th edn, revised) (DSM-IV-TR). APA, 2000

7 First MB, Spitzer RL, Gibbon M, Williams JBW. The Structured Clinical Interview for DSM-III-R personality disorders (SCID-II) Part I: description. J Pers Disord 1995; 9: 83-91.

8 Madge N, Hewitt A, Hawton K, De Wilde EJ, Corcoran P, Fekete S, et al. Deliberate self-harm within an international community sample of young people: comparative findings from the Child \& Adolescent Self-harm in Europe (CASE) Study. J Child Psychol Psychiatry 2008; 49: 667-77.

9 Moran P, Borschmann R, Flach C, Barrett B, Byford S, Hogg J, et al. The effectiveness of joint crisis plans for people with borderline personality disorder: protocol for an exploratory randomised controlled trial. Trials 2010; 11: 18. 
10 Saunders JB, Aasland OG, Babor TF, de la Fuente JR, Grant M. Development of the Alcohol Use Disorders Identification Test (AUDIT): WHO collaborative project on early detection of persons with harmful alcohol consumption. Addiction 1993; 88: 791-804.

11 Zigmund AS, Snaith RP. The hospital anxiety and depression scale. Acta Psychiatr Scand 1983; 67: 361-70.

12 Kapur N, Cooper J, King-Hele S, Webb R, Lawlor M, Rodway C, et al. The repetition of suicidal behavior: a multicenter cohort study. J Clin Psychiatry 2006; 67: 1599-609.

13 Colman I, Newman SC, Schopflocher D, Bland RC, Dyck RJ. A multivariate study of predictors of repeat parasuicide. Acta Psychiatr Scand 2004; 109: 306-12.

14 Hawton K, Rodham K, Evans E, Weatherall R. Deliberate self harm in adolescents: self report survey in schools in England. BMJ 2002; 325 : 1207-11.

15 Horvath AO, Greenberg LS. Development and validation of the Working Alliance Inventory. J Couns Psychol 1989; 36: 223-33.

16 Larsen DL, Attkisson CC, Hargreaves WA, Nguyen TD. Assessment of client/ patient satisfaction: development of a general scale. Eval Program Plan 979; 2: 197-207.

17 Tait L, Birchwood M, Trower P. A new scale (SES) to measure engagement with community mental health services. J Ment Health 2002; 11: 191-8.

18 Tennant R, Hiller L, Fishwick R, Platt S, Joseph S, Weich S, et al. The WarwickEdinburgh Mental Well-being Scale (WEMWBS): development and UK validation. Health Qual Life Outcomes 2007; 5: 63-75.

19 Mundt JC, Marks IM, Shear MK, Greist JM. The Work and Social Adjustment Scale: a simple measure of impairment in functioning. Br J Psychiatry 2002 180: 461-4

20 Gardner W, Hoge SK, Bennett N, Roth LH, Lidz C, Monahan J, et al. Two scales for measuring patients' perceptions for coercion during mental hospital admission. Behav Sci Law 1993; 11: 307-21.

21 Kind P. The EuroQol instrument: an index of health related quality of life. In Quality of Life and Pharmacoeconomics in Clinical Trials (2nd edn) (ed. B Spiker). Lippincott-Raven, 1996.

22 Byford S, Knapp M, Greenshields J, Ukoumunne OC, Jones V, Thompson SG et al. Cost-effectiveness of brief cognitive behaviour therapy versus treatment as usual in recurrent deliberate self-harm: a decision-making approach. Psychol Med 2003; 33: 977-86.
23 National Institute for Health and Clinical Excellence. Guide to the Methods of Technology Appraisal. NICE, 2008.

24 Tyrer $\mathrm{P}$, Thompson G, Schmidt U, Jones V, Knapp M, Davidson K, et al. Randomized controlled trial of a brief cognitive behaviour therapy versus treatment as usual in recurrent deliberate self-harm: the POPMACT study. Psychol Med 2003; 33: 969-76.

25 Barber JA, Thompson SG. Analysis of cost data in randomised trials: an application of the non-parametric bootstrap. Stat Med 2000; 19: 3219-36.

26 Efron B, Tibshirani RJ. An Introduction to the Bootstrap. Chapman Hall 1993.

27 Bateman A, Fonagy P. Randomized controlled trial of outpatient mentalization-based treatment versus structured clinical management for borderline personality disorder. Am J Psychiatry 2009; 166: 1355-64.

28 Linehan MM, Armstrong HE, Suarez A, Allmon D, Heard HL. Cognitivebehavioral treatment of chronically parasuicidal borderline patients. Arch Gen Psychiatry 1991; 48: 1060-4.

29 Bateman AW, Fonagy P. Effectiveness of psychotherapeutic treatment of personality disorder. Br J Psychiatry 2000; 177: 138-43.

30 Campbell MK, Snowdon C, Francis D, Elbourne D, McDonald AM, Knight R et al. Recruitment to randomised trials: strategies for trial enrolment and participation study. The STEPS study. Health Technol Assess 2007; 11: iii, ix-105.

31 Bateman A, Fonagy P. Effectiveness of partial hospitalization in the treatment of borderline personality disorder: a randomized controlled trial. Am J Psychiatry 1999; 156: 1563-9.

32 Borschmann R, Hogg J, Phillips R, Moran P. Measuring self-harm in adults: a systematic review. Eur Psychiatry 2011; 27: 176-80.

33 Evans C, Crawford B. Patient self-reports in pharmacoeconomic studies: their use and impact on study validity. Pharmacoecon 1999; 15: 241-56.

34 Giesen-Bloo J, Van Dyck R, Spinhoven P, Van Tilburg W, Dirkson C, Van Asselt $\mathrm{T}$, et al. Outpatient psychotherapy for borderline personality disorder: randomized trial of schema-focused therapy vs transferencefocused psychotherapy. Arch Gen Psychiatry 2006; 63: 649-58.

35 Henderson C, Flood C, Leese M, Thornicroft G, Sutherby K. Views of service users and providers on joint crisis plans. Soc Psychiatry Psychiatr Epidemiol 2008; 44: 369-76.

36 Kim MM, Van Dorn RA, Scheyett AM, Elbogen EE, Swanson JW, Swartz MS et al. Understanding the personal and clinical utility of psychiatric advance directives: a qualitative perspective. Psychiatry 2007; 70 $19-29$.

\section{poem}

\section{A Message}

Olive M. Ritch

One of the best of minds

destroyed by dementia

does not howl on her knees

in the street, does not masturbate

in the magnolia living-room,

is not dragged off the roof-top,

naked; no, she leaves a message

on her daughter's answer-phone

saying: there's an echo,

an echo in my head.

Olive M. Ritch is currently completing a PhD in creative writing (on the theme of memory) at the University of Aberdeen This poem is from The Hippocrates Prize 2011, published by The Hippocrates Prize in association with Top Edge Press. (c) Olive M. Ritch.

Chosen by Femi Oyebode. 